\title{
Mortality Predictors of Snake Bite Envenomation in Southern India-A Ten-Year Retrospective Audit of 533 Patients
}

\author{
Suresh David • Sarah Matathia - Solomon Christopher
}

Published online: 11 January 2012

(C) American College of Medical Toxicology 2012

\begin{abstract}
Snake bite incidence is highest in Asia and subSaharan Africa. This retrospective audit of 533 adult patients, who had presented to the Emergency Department, collates clinical features, effect of pharmacologic interventions and the risk factors that influence morbidity and mortality. Dual toxicity, neurological and haematological, was observed in $30.4 \%$ of patients. Laboratory evidence of haematotoxicity was demonstrated in 314 (58.9\%) and 40\% demonstrated clinical evidence of bleeding. However, $7.3 \%$ of these patients did not have laboratory evidence of bleeding disorder $(p<$ 0.001 ). Conversely, $60 \%$ did not have clinical evidence of bleeding, but demonstrated laboratory evidence of abnormal parameters. Acute kidney injury (AKI) was evident in $28 \%$ of patients and $15.3 \%$ required haemodialysis. About 25\% with no haematotoxicity showed evidence of AKI. The majority received 6-12 vials of poly-valent anti-snake venom. Hypersensitivity reaction rate was $8 \%$ and predominantly anaphylactoid in nature. The length of hospital stay ranged from 2 to 28 days and $20 \%$ required mechanical ventilation. Overall mortality rate was $7.5 \%$ with significant association to $\mathrm{AKI}$, haematotoxicity and assisted ventilation. The mortality rate was $18 \%$ in patients with pre-hospital delay more than $24 \mathrm{~h}$, as against 5\% when admitted within the above specified period $(p=<0.001)$. The strength of this study is the accrued information
\end{abstract}

S. David $(\bowtie)$

Department of Emergency Medicine, Christian Medical College,

Vellore 632004, India

e-mail: suresh.david@cmcvellore.ac.in

\section{S. Matathia}

University of California,

San Francisco, CA 94110, USA

S. Christopher

Department of Clinical Epidemiology, Christian Medical College,

Vellore 632002, India of over a period of 10 years of snake-bite management through the Emergency Department of a university hospital setting. The limitations are the retrospective study design and the rejection percentage of $15.5 \%$ due to insufficient information from the total chart pool.

Keywords Snake bite P Polyvalent ASV · Anti-snake venom $\cdot$ Mortality

\section{Introduction}

Snake bite injury data published by the World Health Organization estimates an incidence of five million snakebite cases worldwide each year. Reportedly, about 200,000 persons fall prey to snake bite per year in India and 50,000 of them die every year [1]. The highest incidence of snake bites is in South Asia, Southeast Asia and sub-Saharan Africa and caused by elapids (cobras, kraits, mambas, etc.) and vipers, and in some regions, sea snakes. This retrospective analysis of a decade of information collected from a University teaching hospital was intended for comprehensive understanding of the epidemiologic and clinical features of snake bite envenomation in South India.

\section{Aims and Objectives}

- To study the epidemiology, and the effect of pharmacologic interventions pertaining to snake bite

- To analyse the association between various risk factors and their influence on the final outcome

Study Design Retrospective chart review 
Study Population All patients were 18 years and older who had presented to the Emergency Department with history of snake bite over a period of 10 years, 1997-2007.

Exclusion Criteria None.

Outcome measures:

1. Mortality: Died prior to discharge from hospital

2. Morbidity: Renal dysfunction, tissue loss, limb amputation, etc.

3. Length of stay: Number of days admitted to the hospital

\section{Methodology}

Relevant data were collected from medical records of the study population on the Chart Review Forms. Details included Epidemiologic Information, snake bite details, symptomatology and resuscitation requirements, in addition to indications for anti-snake venom (ASV) administration, based on clinical and laboratory investigations. The patient's progress was followed through the entire period of hospital stay and the outcome documented.

Ethical Considerations None. All patient identification will be de-identified per protocol to comply with research standards.

Sample Collection The sample size of 533 patients was derived from a total number of 628 charts (Fig. 1). Statistical analyses were performed using SPSS 11.0 for windows. Comparisons of continuous variables were assessed using $t$ test and categorical variables were analysed using chi-square test. Variables that were significantly related to mortality at the

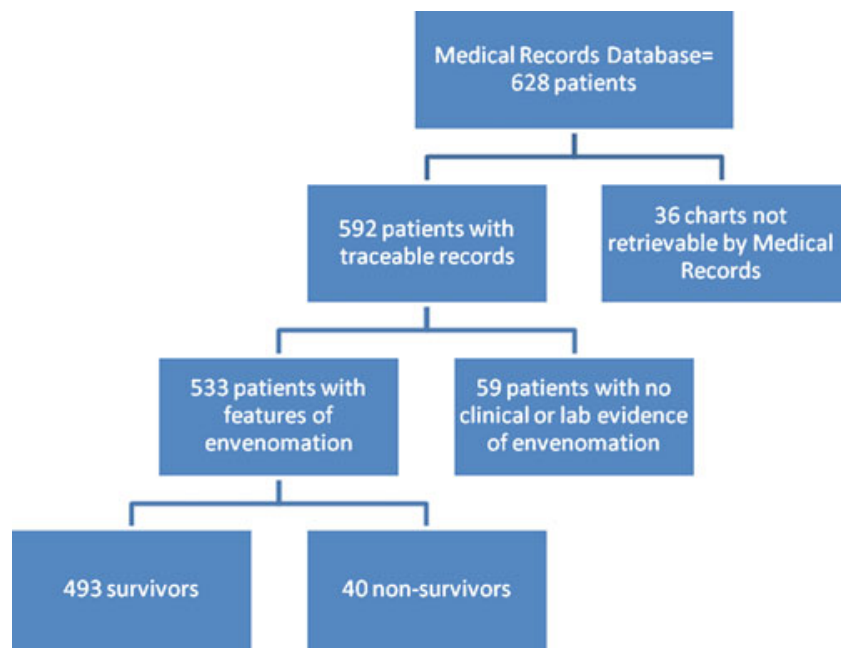

Fig. 1 Sample size derivation
Table 1 Baseline characteristics

\begin{tabular}{|c|c|c|}
\hline \multirow[t]{3}{*}{ Demography } & Age (years) & $32 \pm 15.9$ \\
\hline & Male & $370(69.4 \%)$ \\
\hline & Hospitalisation within $24 \mathrm{~h}$ & $409(76.7 \%)$ \\
\hline \multirow[t]{3}{*}{ Bite site } & Upper limb & 111 \\
\hline & Lower limb & 382 \\
\hline & Trunk and head & 20 \\
\hline \multirow[t]{13}{*}{ Clinical Findings } & Heart rate/min & $93.6 \pm 21.3$ \\
\hline & $\begin{array}{l}\text { Mean systolic } \\
\text { pressure - } \mathrm{mmHg}\end{array}$ & $93.8 \pm 15.6$ \\
\hline & Respiratory rate/min & $25.5 \pm 7.7$ \\
\hline & $\begin{array}{l}\text { Abnormal } \\
\text { pulse-oximetry } \%\end{array}$ & $86(16.1 \%)$ \\
\hline & Loss of consciousness & $87(16.3 \%)$ \\
\hline & Vomiting & $195(36.6 \%)$ \\
\hline & Ptosis & $332(62.3 \%)$ \\
\hline & Diplopia & $92(17.3 \%)$ \\
\hline & Ophthalmoplegia & $134(25.1 \%)$ \\
\hline & Dyspnoea & $146(27.4 \%)$ \\
\hline & Dysarthria & $74(13.9 \%)$ \\
\hline & Muscle weakness & $145(27.2 \%)$ \\
\hline & Bleeding at bite site & $142(26.6 \%)$ \\
\hline \multirow[t]{5}{*}{ Laboratory Findings } & Haematuria & $92(17.3 \%)$ \\
\hline & Prolonged PT & $253(47.5 \%)$ \\
\hline & Prolonged INR & $198(37.1 \%)$ \\
\hline & Prolonged PTT & $173(32.5 \%)$ \\
\hline & Leucocytosis & $16.1(1.1-72.8)$ \\
\hline \multicolumn{3}{|l|}{ Toxicity } \\
\hline & Nil & 26 \\
\hline & Haemato-toxicity & 152 \\
\hline & Neurotoxicity & 193 \\
\hline & Dual toxicity & 162 \\
\hline \multicolumn{3}{|l|}{ Management } \\
\hline \multirow[t]{9}{*}{ ASV Reaction } & & $41(7.7 \%)$ \\
\hline & Anaphylaxis & $12(2.3 \%)$ \\
\hline & Anaphylactoid reactions & $29(5.4 \%)$ \\
\hline & Nil reaction & $491(92.3)$ \\
\hline & Mechanical ventilation & $105(19.7 \%)$ \\
\hline & Inotrope support & $48(9 \%)$ \\
\hline & Blood transfusion & $42(7.9 \%)$ \\
\hline & FFP transfusion & $45(8.4 \%)$ \\
\hline & Surgical intervention & $19(3.6)$ \\
\hline \multirow[t]{4}{*}{ Outcome } & Non-envenomated cases & $59(11.064 \%)$ \\
\hline & Acute kidney injury & $143(26.8 \%)$ \\
\hline & Mortality & $40(7.5)$ \\
\hline & $\begin{array}{l}\text { Mean length of hospital } \\
\text { stay (days) }\end{array}$ & $4(1-70)$ \\
\hline
\end{tabular}

univariate analyses $(p<0.1)$ were included in a multivariable logistic regression model. Unadjusted and adjusted odds ratios are presented for all the risk factors for mortality. 
Fig. 2 Seasonal variation in snake bite incidence

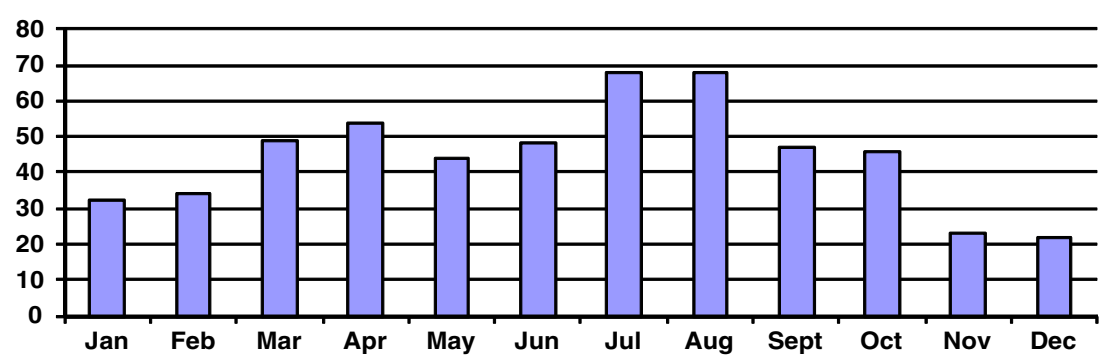

\section{Results}

The demography of the study population, bite site, clinical findings, investigations, toxicity and ASV reactions in addition to the final outcome are depicted in Table 1. Snake bite was noted pre-dominantly in the rural agricultural population of India, with male predominance. The main species which had been identified in the study area are Russell's viper (Daboia russelii), Common Cobra (Naja naja) and Common Krait (Bungarus caeruleus). The bite environment was recorded in $43 \%$ of cases and it occurred outdoors-while working or sleeping in the open, during summer months. Seasonal variation similar to our observation has been noted elsewhere [2] with the predominant incidence being in the rainy season-June to September (Fig. 2).

Bite site was mostly in the lower limbs. Although about one fourth of the patients or their relatives volunteered to identify the species of the snake, its reliability, however, could not be confirmed. About three fourth of the patients sought first aid in local clinics and peripheral hospitals prior to definitive treatment in the University hospital (research setting). Most of the cases were admitted within $24 \mathrm{~h}$ of the bite. Morbidity included cellulitis, neurotoxicity and haematotoxicity. Dual toxicity was observed in $30.4 \%$ of patients.

About $40 \%$ demonstrated clinical evidence of bleeding. However, $7.3 \%$ of these patients did not have laboratory

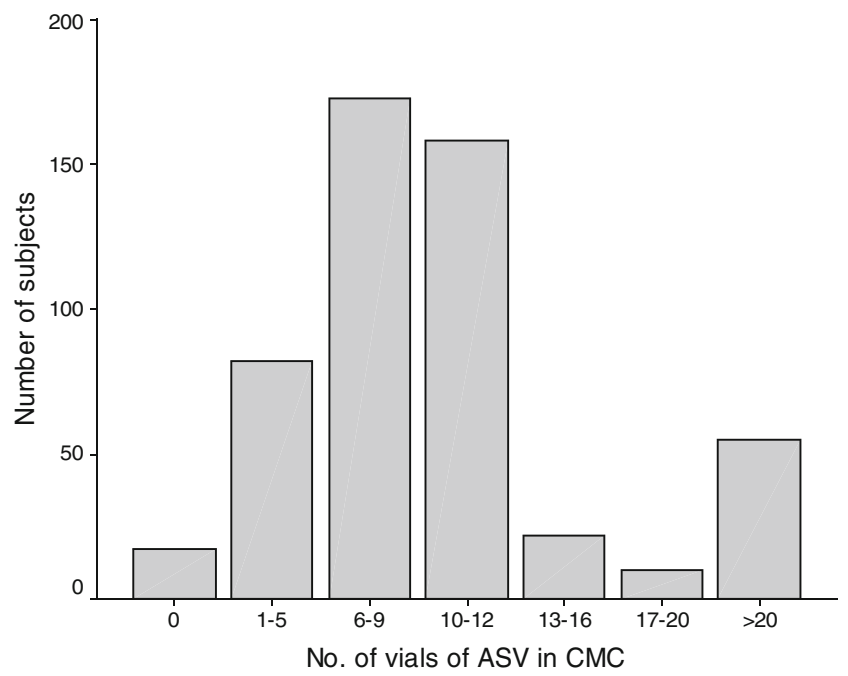

Fig. 3 Anti-snake venom therapy in the setting of the study evidence of bleeding disorder. This is statistically significant $(p<0.001)$. Conversely, $60 \%$ of patients did not have clinical evidence of bleeding, but demonstrated laboratory evidence of abnormal parameters. Laboratory evidence of acute renal failure was noted in $28 \%$ of patients with clinical signs of haematotoxicity and $15.3 \%$ required haemodialysis. Laboratory evidence of haematotoxicity (defined in our study as any of the following: PT>13.2 s, PTT>37.2 s, CT>20 min and BT> $7 \mathrm{~min}$ ) was demonstrated by 314 patients (58.9\%). About $25 \%$ with no haematotoxicity showed evidence of abnormal renal function. Abnormal platelet count (defined in our study as $<60,000$ ) was noted in $16 \%$ and about $81 \%$ demonstrated leucocytosis (WBC>20,000), ranging up to $40,000 / \mathrm{mm}^{3}$.

All the 533 cases received polyvalent ASV which is effective against all the four common species, which are as follows: Russells viper (D. russelii), Common Cobra ( $N$. naja), Common Krait (B. caeruleus) and Saw Scaled viper (Echis carinatus). There are no currently available monovalent ASVs primarily because there are no objective means of identifying the snake species, in the absence of the dead snake. It would be impossible for the physician to determine which type of monovalent ASV to employ in treating the patient. The hypersensitivity reaction rate was about $8 \%$ and was predominantly minor anaphylactoid in nature-pruritus, hypotension, urticarial rash, syncope, fever, etc. Dosage data were available for 517 patients and the majority received 6-12

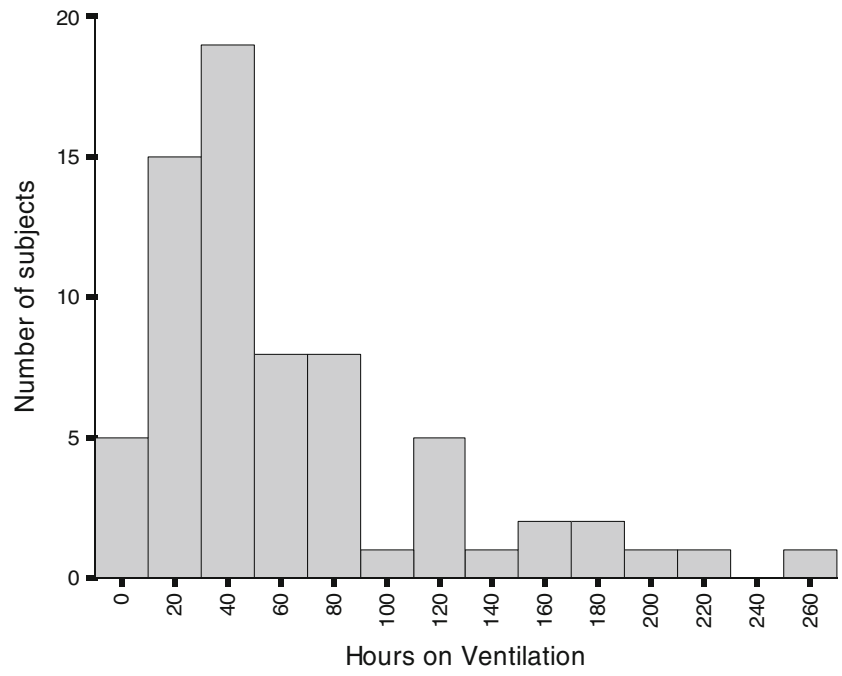

Fig. 4 Hours of ventilation in the ICU 


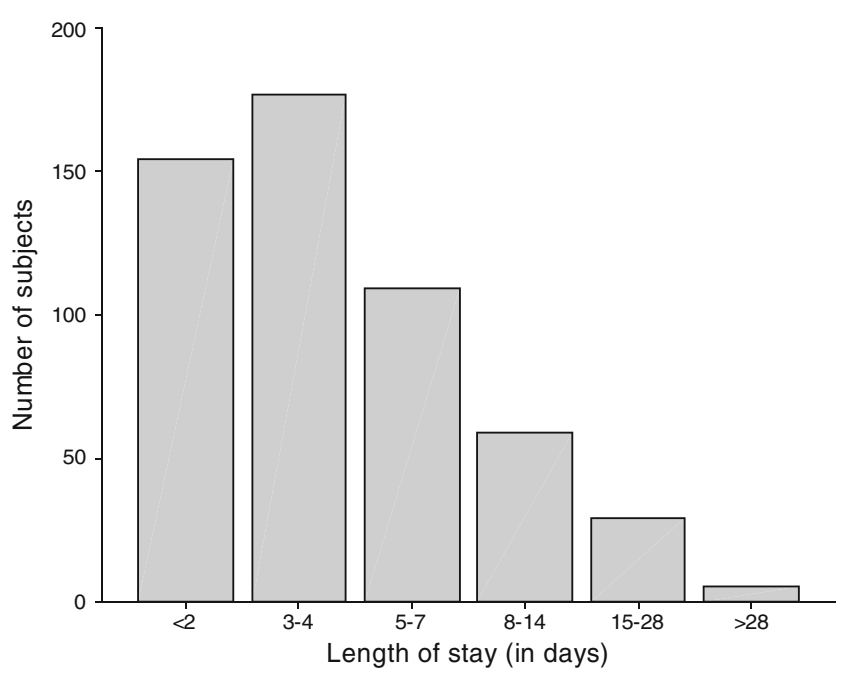

Fig. 5 Length of hospital stay (in days)

vials of ASV (Fig. 3). About 9\% of the patients required inotropic support and $8 \%$ needed blood transfusion. Fresh frozen plasma was needed in $8 \%$ of patients. Tetanus toxoid was administered in $95 \%$ of patients. Surgical intervention included debridement with skin or flap cover, incision and drainage. One patient required amputation.

Admission to the intensive care unit was required in $40 \%$ of the total population. About $20 \%$ required mechanical ventilation, which extended up to 10 days (Fig. 4). The indications were respiratory insufficiency, due to neuro-toxicity. Of these, 33\% expired. Of the remaining patients who did not require the same, $1.5 \%$ died, thus demonstrating statistical significance in mortality in relationship with assisted ventilation $(p=<0.001)$. Length of hospital stay ranged from 2 to 28 days (Fig. 5). Morbidity included renal failure, intra-cranial bleeds, post-anoxic encephalopathy, limb contractures, acute coronary syndrome, amputations, etc. (Table 2). The overall mortality rate was $7.5 \%$. Multiple logistic regression analysis on all factors which influenced mortality at the univariate level demonstrated a significant association between acute kidney injury (AKI), haematotoxicity and assisted ventilation with respect to mortality. Patients with AKI demonstrated a mortality of $21 \%$ as against $3 \%$ of those who died and did not have AKI $(p=<0.001)$.

Age group analysis demonstrated maximum mortality in patients above $65(20 \%)$ and $<10$ years $(11 \%)$, thereby including the extremes of age. There was no association age and delay to presentation against intubation and acute kidney injury. Patients in whom there was pre-hospital delay more than $24 \mathrm{~h}$, the mortality was $18 \%$ as against $5 \%$ in those who were admitted within the above specified period $(p=<0.001)$. Significant statistical relationship $(p=<0.001)$ was observed in the total number of 20 patients $(23 \%)$ who presented with loss of consciousness at the time of presentation to the Emergency Department who expired subsequently.

\section{Discussion}

The strength of this study is the accrued information available over a decade of snake bite management in a university hospital setting, which endeavours to practice evidence-based medicine. India remains amongst the group of countries with the highest mortality [3]. Globally, usage of monovalent ASV is the preferred mode of treatment. However, In India and the neighbouring countries, due to the usage of polyvalent vaccine, species identification is not of critical clinical value. Snake identification was recorded in one fourth of the subjects, since visibility and fear were significant obstacles, as observed elsewhere [2]. This impedes the investigation of the epidemiology of speciesspecific biological behaviour of envenomation. Further, species that dominate the snake bite population differ according to geography $[4,5]$. Of recent interest is the discovery of another species, the hump-nosed pit viper (Hypnale hypnale), also capable of causing lethal envenomation [6]. This necessitates the development of a more inclusive polyvalent vaccine, since the present drug is exclusively for the Indian cobra ( $N$. naja), the common krait (B. caeruleus), the Russell's viper ( $D$. russelii) and the saw-scaled viper (E. carinatus).
Table 2 Risk factors and morbidity profile due to snake bite

${ }^{\text {a }}$ Statistically significant at $5 \% \alpha$ level

${ }^{\mathrm{b}}$ Reference category

\begin{tabular}{|c|c|c|c|c|}
\hline \multirow[t]{2}{*}{ Risk factors } & \multicolumn{2}{|l|}{ Mortality } & \multicolumn{2}{|l|}{ Odds ratio } \\
\hline & Alive & Dead & Unadjusted & Adjusted \\
\hline Acute renal failure & $113(22.9 \%)$ & $30(75 \%)$ & 10.1 & $5.2^{\mathrm{a}}$ \\
\hline Hospitalization within $24 \mathrm{~h}$ & $389(80.9 \%)$ & $20(50 \%)$ & 4.2 & 1.1 \\
\hline Ventilation & $71(14.4 \%)$ & $34(85 \%)$ & 33.7 & $46.7^{\mathrm{a}}$ \\
\hline Presence of haematotoxicity & $27(5.5 \%)$ & $19(47.5 \%)$ & 15.6 & 2.8 \\
\hline Platelets $<60$ & $25(6.8 \%)$ & $14(41.2 \%)$ & 12.1 & 5.1 \\
\hline $60-100$ & $19(5.2 \%)$ & $5(14.7 \%)$ & 5.7 & 1.8 \\
\hline$>100^{\mathrm{b}}$ & $323(88 \%)$ & $15(44.1 \%)$ & & \\
\hline $\mathrm{WBC} \leq 11,000$ & $93(21.6 \%)$ & $7(18.9 \%)$ & 1.2 & 0.9 \\
\hline
\end{tabular}


One of the challenges in envenomation medicine is the inability to quantify venom dose, thus making treatment protocols arbitrary. The quantity of venom that can be extracted from the Russell's viper varies significantly and depends on the size of the snake [7]. The range of venom injected was shown to be 5-147 mg. This would imply a maximum ASV dose of around 25 vials [8]. ASV is an expensive commodity and is of perennial short supply. Therefore it needs to be used judiciously. Randomised control trials in two independent settings which administered high dose ASV to one study arm and low dose ASV to the other, concluded that there was no statistical difference between the two arms in terms of mortality, length of stay or supportive measures $[9,10]$. Dosage requirements and recommendations have varied significantly - from an average of 51.2 vials for cobra bites and 31 vials for viper bites [11]. Guidelines based on Russell's Viper venom recommended the administration of 10 units of ASV, and only if the patient exhibits signs or symptoms of systemic or severe local envenomation [12]. The biological behaviour and benefit of low-dose ASV therapy have been observed to exhibit an overall mortality rate of $7.5 \%$, following the low-dose protocol. This is encouraging and provides the impetus for further investigation. One particular study of 277 cases of snake bite envenomation examined predictors of mortality - vomiting (RR-0.51), neurotoxicity (RR-3.15) and creatinine elevation (RR1.35) [13]. This study also indicated that acute renal failure and the need for ventilation bear significance in predicting mortality.

It has been recommended by the WHO in 2005 that snake bite should be formally recognised as an important occupational disease and should be made a specific notifiable disease in all countries in the South East Asian region [14]. It is also recommended that education and training on snake bite should be included in the curriculum of medical schools. This study has indicated that bites were more common when working and sleeping outdoors. Therefore, there is significant scope for public health intervention, i.e. community education, awareness about snake bite, wearing shoes and sleeping indoors as an effective method to mitigate the consequences of envenomation.

This retrospective chart review has its limitations since the retrospective information gleaned was from 533 patients over 10 years, with a rejection percentage of $15.5 \%$ due to insufficient information from the total chart pool. Moreover, the selection bias due to a singlecentre data is justifiable. Furthermore, no prior baseline information about the study population was available. For example, anaemia was noted in one third of the study population. However, no conclusion could be drawn from this observation, since their baseline haemoglobin is unknown.
Wound aspirate, serum and urine can be subjected to enzyme-linked immunoassay (ELISA) and radioimmunoassay for detection of snake venom and venom antibodies [15]. They can detect values as low as $0.5 \mathrm{ng}$ of venom. Current ELISA systems involving snake venoms have low specificity, and most cannot reliably differentiate venoms of related snakes. Nonspecific reactions and cross-reactivity are unacceptably high. However, there is scope for further development in this field.

\section{Conclusion}

Published literature depicts significant variation that exists between regions of India and in the Asian sub-continent. There is dearth of collective evidence for clear pathways to be determined to predict the outcome of snake envenomation in the above geographical area. The objective of this exercise - the largest case series of its kind - was to collate a decade of experience from best practice standards of a University teaching medical institute of repute, to facilitate comprehensive understanding of the disease behaviour and to refine patient care guidelines.

\section{References}

1. http://www.who.int/neglected diseases/diseases/snakebites/en/ index.html. Last accessed on June 3, 2010.

2. Brunda G, Sashidhar RB (2007) Epidemiological profile of snakebite cases from Andhra Pradesh using immunoanalytical approach. I. Indian J Med Res 125(5):661-668

3. Simpson ID (2007) Snakebite management in India, the first few hours: a guide for primary care physicians. J Indian Med Assoc 105(6):324-326

4. Kirte RC, Wahab SN, Bhathkule PR (2006) Record based study of snake bite cases admitted at Shri Vasantrao Naik Government Medical College \& Hospital, Yavatmal (Maharashtra). Indian J Publ Health 50(1):35-37

5. Bhardwaj A, Sokhey J (1998) Snake bites in the hills of north India. Nat Med J India 11(6):264-265

6. Simpson ID, Norris RL (2007) Snakes of medical importance in India: is the concept of the "Big 4" still relevant and useful? Wilderness Environ Med 18(1):2-9

7. Tun P, Cho KA (1986) Amount of venom injected by Russell's viper (Vipera russelli). Toxicon 24(7):730-733

8. Simpson ID (2007) The paediatric management of snakebite: the National Protocol. Ind Ped 44(3):173-176

9. Paul V, Pratibha S, Prahlad KA et al (2004) High-dose anti-snake venom versus low-dose anti-snake venom in the treatment of poisonous snake bites - a critical study. J Assoc Phys India $52: 14-17$

10. Tariang DD, Philip PJ, Alexander G, Macaden S, Jeyaseelan L, Peter JV, Cherian AM (1999) Randomized controlled trial on the effective dose of anti-snake venom in cases of snake bite with systemic envenomation. J Assoc Phys India 47 (4):369-371 
11. Bawaskar HS, Bawaskar PH, Punde DP, Inamdar MK, Dongare RB, Bhoite RR (2008) Profile of snakebite envenoming in rural Maharashtra, India. J Assoc Phys India 56:88-95

12. Ghosh S, Maisnam I, Murmu BK, Mitra PK, Roy A, Simpson ID (2008) A locally developed snakebite management protocol significantly reduces overall anti-snake venom utilization in West Bengal, India. Wilderness Environ Med 19(4):267274

13. Kalantri S, Singh A, Joshi R, Malamba S, Ho C, Ezoua J, Morgan $M$ (2006) Clinical predictors of in-hospital mortality in patients with snake bite: a retrospective study from a rural hospital in central India. Trop Med Int Health 11(1):22-30

14. David AW (2005) Guidelines for the clinical management of snake-bites in the south-east Asia region. World Health Organization, Regional Office for South East Asia, New Delhi pp 1-67. http://www.searo.who.int/LinkFiles/SDE_mgmt_snake-bite.pdf. Last accessed on June 15, 2010

15. Selvanayagam ZE, Gopalakrishnakone P (1999) Tests for detection of snake venoms, toxins and venom antibodies: review on recent trends (1987-1997). Toxicon 37(4):565-586 